\title{
Lipase-catalyzed dimethyl adipate synthesis: response surface modeling and kinetics
}

\begin{abstract}
Dimethyl adipate (DMA) was synthesized by immobilized Candida antarctica lipase Bcatalyzed esterification of adipic acid and methanol. To optimize the reaction conditions of ester production, response surface methodology was applied, and the effects of four factors namely, time, temperature, enzyme concentration, and molar ratio of substrates on product synthesis were determined. A statistical model predicted that the maximum conversion yield would be $97.6 \%$, at the optimal conditions of $58.5^{\circ} \mathrm{C}, 54.0 \mathrm{mg}$ enzyme, $358.0 \mathrm{~min}$, and 12:1 molar ratio of methanol to adipic acid. The R2 (0.9769) shows a high correlation between predicted and experimental values. The kinetics of the reaction was also investigated in this study. The reaction was found to obey the ping-pong bi-bi mechanism with methanol inhibition. The kinetic parameters were determined and used to simulate the experimental results. A good quality of fit was observed between the simulated and experimental initial rates.
\end{abstract}

Keyword: Biocatalysis; Enzymatic synthesis; Esterification; Kinetics; Optimization 